\title{
Chirality and Helicity of Poly-Benzyl-L-Glutamate in Liquid Crystals and a Wave Structure that Mimics Collagen Helicity in Crimp
}

\author{
Benedicto de Campos Vidal*, Maria Luiza Silveira Mello \\ Department of Cell Biology, Institute of Biology, UNICAMP, \\ 13083-970 Campinas - SP, Brazil
}

Received: November 18, 2000; Revised: June 23, 2001

\begin{abstract}
Ideal biocompatible polymers must show a mimetic superstructure with biological supra-organization. Collagen-rich structures like tendons and ligaments are materials with various levels of order, from molecules to bundles of fibers, which affect their biomechanical properties and cellular interactions. Poly-benzyl-L-glutamate (PBLG) displaying helicity was used here to test the development of wave-like structures as those occurring in collagen fibers. Birefringence of PBLG under various crystallization conditions was studied with a $\lambda / 4$ compensator according to Sénarmont. Qualitative observations were plainly sufficient to conclude that the PBLG fibrils were supra-organized helically as a chiral object. During crystallization stretched PBLG formed a helical superstructure with characteristic striation resembling waves (crimp). Supported by optical anisotropy findings, a twisted grain boundary liquid crystal type is proposed as a transition phase in the formation of the PBLG chiral object. A similarity with the wavy organization (crimp) of collagen bundles is proposed.
\end{abstract}

Keywords: poly-benzyl-L-glutamate, wave-like superstructure, liquid crystal, optical anisotropy

\section{Introduction}

Many types of molecules have been studied for their possible use as thin films with polar order and piezo-pyroelectrical properties, particularly in photo-optical and electromagnetic applications and in the imaging of spatially resolved chemical libraries ${ }^{1-7}$. The most important properties in producing these supramolecular constructs are their mesophasic characteristics of self-assembly and chirality. These make it possible to examine the structure-function relationships in depth, particularly the relationship between optical characteristics, molecular order and the various types of birefringence.

The chirality of certain macromolecules allows the formation of helical superstructures ${ }^{6,8}$. Actually, non-chiral molecules can also generate chiral objects, provided that polar order and geometry of such molecules are present, e.g. rod-like/banana-like molecules are required to construct a chiral layer, the chirality of the layer acting as a chiral object itself that can in turn produce a helical superstructure 9 .

*e-mail: vidal@unicamp.br
Atomic force microscopy suggested that collagen molecules exist as a chiral construct in collagen bundles ${ }^{10,11}$. To study similarities with collagen, the use of poly-benzyl-Lglutamate (PBLG) as a model (a reducionist approach) could shed some light to this question.

The polypeptide polyglutamic acid and its benzyl ester, poly-benzyl-L-glutamate(PBLG), have been used as a model for studying polar order, self-assembly, helicity, piezo-pyroelectric properties and for determining the conditions that lead to pyro-piezoelectricity ${ }^{2,12,13}$. It has been demonstrated that the superstructure of PBLG fibers has crystalline and textural birefringence ${ }^{14}$. Based on these data it is possible to determine that $58.8 \%$ of the total birefringence was accounted by crystalline birefringence. These properties are important when seeking to correlate the presence of waves (crimp) in the superstructure of PLBG with the molecule's chirality and order.

In this study, mesophase chiral polymers of PBLG were examined for optical anisotropies which would suggest a helical supra-organization. The occurrence of wave-like structures (crimp) caused by mechanical stretching on the 
PBLG mesophase during crystallization are assumed to be related to the helical chiral PBLG characteristics and mimic those previously reported for collagen crimp ${ }^{15,16}$.

\section{Material and Methods}

A viscous solution of poly-(-benzyl-L-glutamate) (PBLG) was prepared by dissolving $100 \mathrm{mg}$ of PBLG (Sigma (150-300)x $10^{3}$ ) in $1 \mathrm{~mL}$ of chloroform. Drops of this solution were placed on slides and left to dry. In some cases, the drops were placed close to each other so that their peripheries came into contact. In other cases, the drops were allowed to spread on the surface of bidistilled water on which they dried. Stretching the drops, while evaporating the chloroform, was done using $26 \mathrm{G}$ x $1 / 2$ " needles in order to obtain stretched fibers of various diameters.

Optical anisotropy was assessed using dry as well as Cargille oil-imbibed PBLG. A Zeiss-Pol photomicroscope was used. The $\lambda / 4$ mica plate Sénarmont compensator was used to determine the birefringence signal of the PBLG fibers and detect their orientation in the PBLG superstructures, by placing the Sénarmonts compensator gamma (direction of vibration) parallel to the analyser azimuth (known also as plane of light polarization, PLP). In this case no birefringence of the compensator is introduced. The function of the compensator is to transform helically polarized light emerging from the object into a plane polarized light, which will be compensated by rotating the analyser. The compensation produced quantitative data not used for this work since the qualitative results were plainly sufficient to allow description of the events and to support inferences.

\section{Results}

After drying on slides and on the surface of water, the PBLG solution formed fibrillar films that showed positive birefringence as determined by Sénarmonts compensation.

Isolated drops as well as drops touching each other showed rings of different superstructures, from the periphery directed towards the center of the drop. At the drop periphery or at touching surfaces, a thin fibrillar membrane was seen, the fibrils of which were detected by birefringence and formed a frame structure as shown by using Sénarmont's compensator. The fibrils were intertwined helically at various levels, giving rise to a hierarchy of fibers with increased degrees of packing towards the center of the drop. Details of the coiled coils were observable with Sénarmont's compensation. The various levels of rings exhibited different degrees of helically intertwined fibrils, fibers and bundles of fibers, all of which produced varying degrees of compactness. A very compact ring of fibers was formed in the center of the drop, because of the higher concentration of PBLG (Fig. 1).

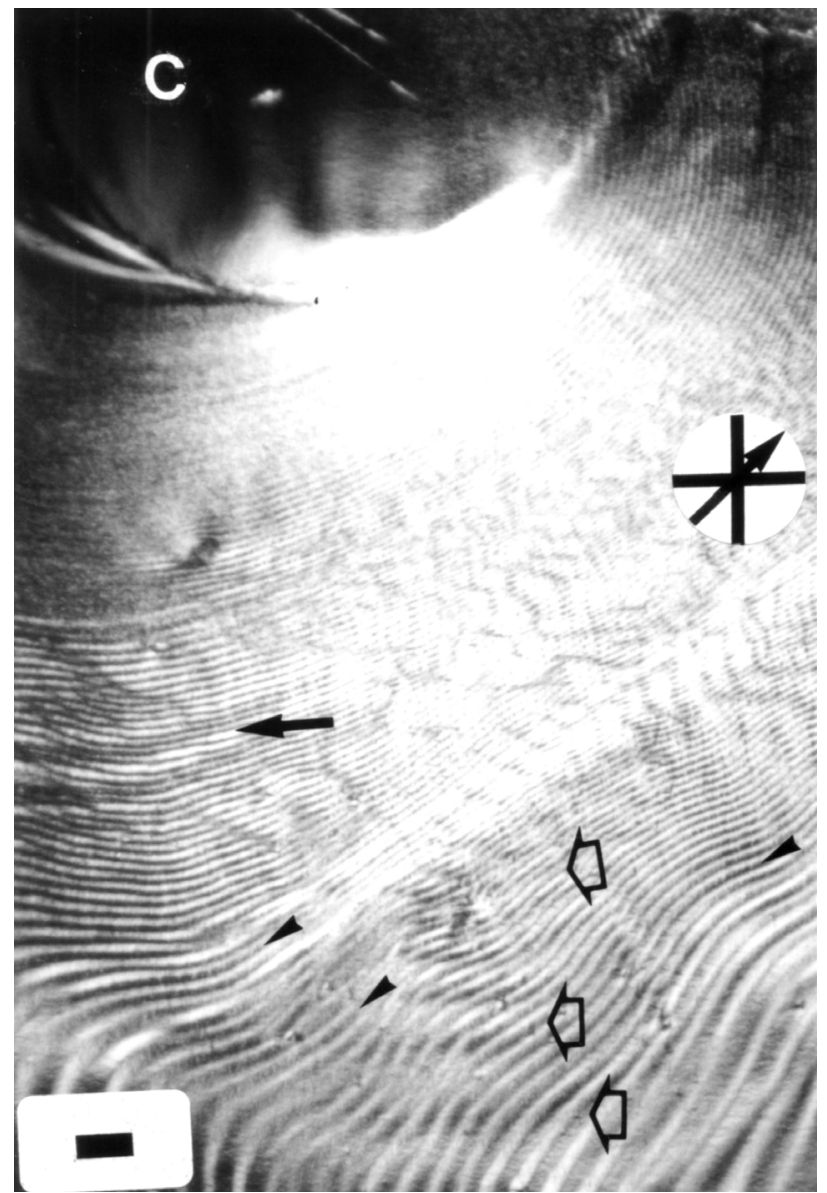

Figure 1. Birefringence image of the superstructure resulted from crystallization of two drops of PBLG solution in contact with each other as seen after Sénarmont's compensation. Compensated columns or cords appear black (arrow head), whereas non-compensated ones appear bright. The alternate compensation of the cords reveals their antiparallel molecular arrangement. The cords were positioned at 45 degrees with respect to the polarizer. The crossed lines indicate polarizer and analyzer azimuths, and the arrow indicates the respective statistical direction of the cord axis. Some degree of intertwining of cords due to changes in their direction is shown (arrow). These changes also reveal undulations (short empty arrow). C, center of one of the drops. Bar, $100 \mu \mathrm{m}$.

Tubular, columnar or cord-like structures were formed when the periphery of two drops touched one another. The resulting birefringent layers were similar (but not identical) to those previously described ${ }^{17,18}$ and resembled DNA cords ${ }^{19}$, being almost parallel one to another along the area of contact between them (Figs. 1 and 2). The direction and orientation of the fibrils in these bands were established by determining the birefringence characteristics. The cord-like structures exhibited antiparallel position-orientation in which one cord-like structure had its birefringence compensated, while the other maintained its birefringence (Figs. 1 and 2). Internally, each cord showed an alternating compensation of the birefringence of the fibrillar components seen as a striated-like aspect since compensated and 


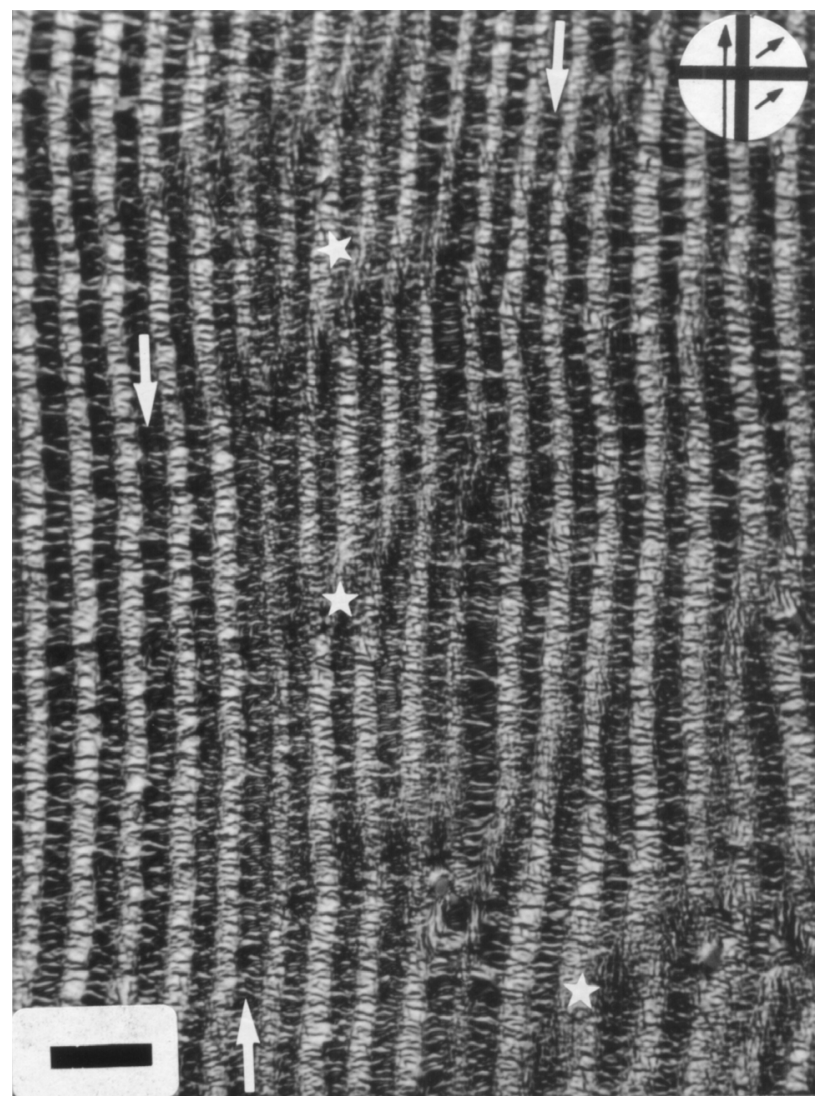

Figure 2. Detail from Fig. 1. The compensated cords are dark (white arrows), and almost parallel to the non-compensated bright birefringent ones. Compensated and non-compensated cords alternate. Where the cords are out of the focalization level, fibrilar components of the cords are seen (white stars). Striations are observed as non-compensated elements in the dark cords. The schema represents the relative orientation of cords that were parallel to the analyzer (large arrow). The small arrows represent the compensated positive birefringent molecules in the cords positioned at 45 degrees from the analyser. Bar, $30 \mu \mathrm{m}$.

non-compensated birefringent fibrillar striations alternate (Fig. 2).

Stretching PBLG liquid crystals during drying revealed a distinct bright birefringence with a wave appearance (crimp) demonstrated with the compensator (Fig. 3A and 3B), indicating the alternating directions of the molecular order of the fibrillar superstructure. The bands also showed areas of fusion essential for maintaining the fitness of the wave (crimp) superstructure.

\section{Discussion}

Chiral molecules forming a molecular assembly have been reported to generate helical superstructures that may be related to the Cotton effect ${ }^{1}$. The report of Akagi and co-workers ${ }^{1}$ is a striking instance of correlation among chirality, birefringence striae or bands and fibrous helical morphologies detected by scanning electron microscopy. Polyglutamic acid in an -helix conformation and PLGA-acridine orange complexes in solution also exihibit a Cotton effect that is comparable to the optical anisotropic properties of PLGA-toluidine blue complexes ${ }^{20}$.

The results of the present study are in agreement with the report quoted above ${ }^{1}$, and clearly establish the hierarchy of helicity from fibrillar membrane structures to twisted coils and then in a final rope or tube-like morphology that reflects helical layers corresponding to helical periods. The stretching caused the formation of striae (bands) as if an helical spring was stretched. These structures form an ordered network superstructure with fibrillar characteristics which is in agreement with reports of a twist-grain-boundary (TGB) phase exhibiting a helical twist ${ }^{21-23}$. TGB phases often occur between smectic and cholesteric or isotropic phases $^{23}$.

The observations in the present study were based on crystallized dry PBLG in which a concentration gradient was formed by rapid chloroform evaporation from the drop meniscus on the surface of the slides. The mechanisms involved in the formation of the rope-like superstructure in the regions where drops contacted at their periphery may be similar to those occurring at cell walls during the formation of equispaced lines parallel to the sides of the cells ${ }^{18}$. Whatever the mechanism, intermolecular forces of the selfassembly process, polarity and the helical geometry of the molecules must be considered.

The supramolecular organization involved is the source of textural (form or structural) birefringence, and obeys physical refraction and birefringence theories ${ }^{24-27}$. The textural aspect depends on the molecular geometry, on the partial volume and/or the aggregation and orientation of the molecules contained in the fibers or bundles. The exhibited textural/form birefringence ${ }^{14}$ appears by virtue of the helical arrangement of the constituent molecules ${ }^{21}$ and their geometry. The wave-like structure appearing under stress/strain is related to the elastic phase behaviour of a TGB, including zig-zag defect formation ${ }^{28}$. Accurate, controlled conditions, established a relationship between shear and the striations formed perpendicular to the direction of shear $^{29}$. The results obtained here agreed with the latter study. The differences between these two reports may reflect the tougher conditions of shear, higher concentration of PBLG, type of solvent, the conditions of chloroform evaporation, and the use of a fine needle to stretch the solution during solvent evaporation.

The piezoelectric effect reported for PBLG films, considered as being a prototype material ${ }^{13}$ derives from the characteristics and properties of the ordered superstructure described above. The organization of the PBLG molecules resembles that of the helical, wave morphology of collagen bundles (crimp) ${ }^{15,16}$. Atomic force microscopy has confirmed the hierarchical supramolecular organization of collagen in a helical coil of coils ${ }^{3,11}$. 

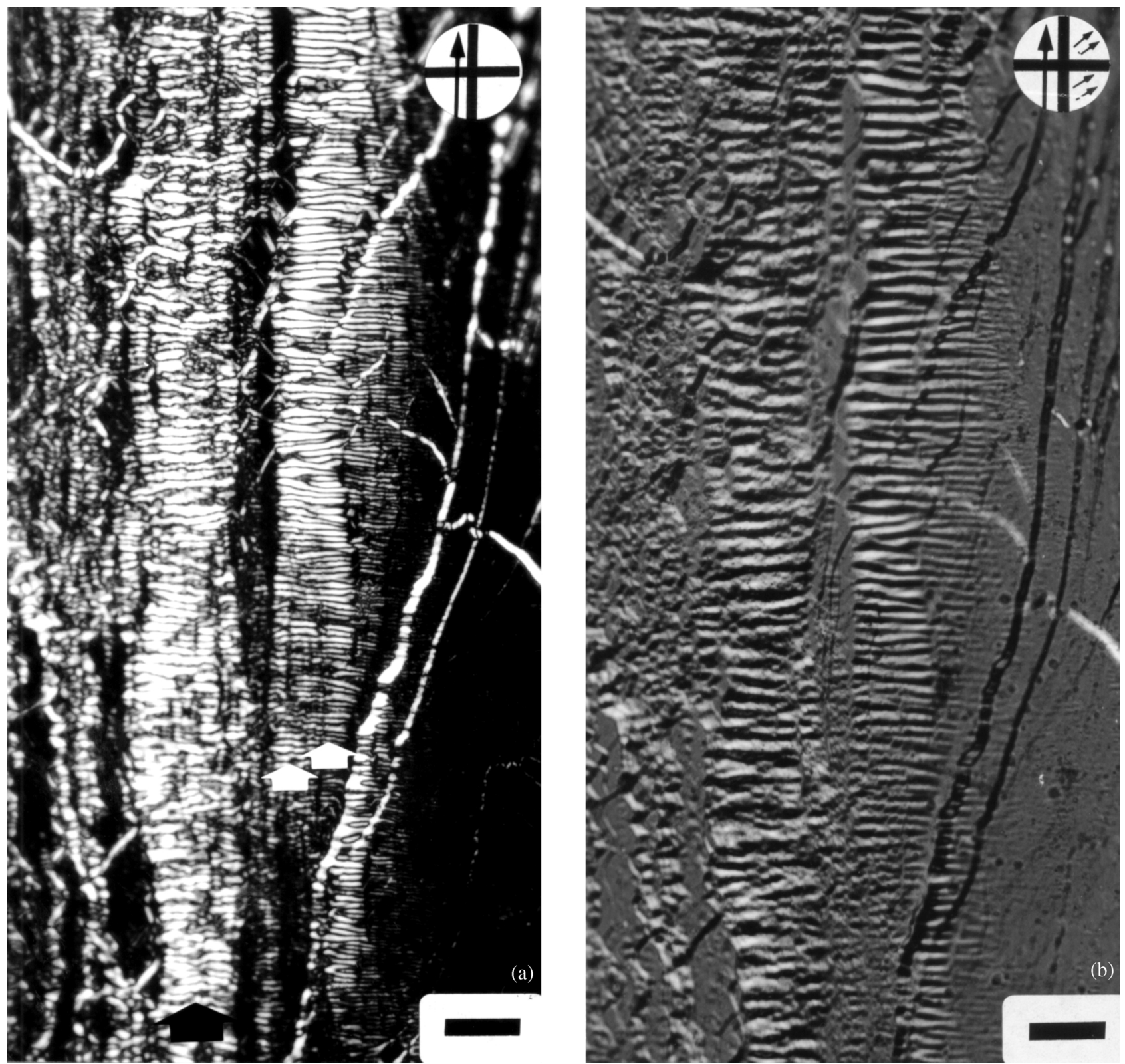

Figure 3. Birefringent images obtained after stretching the PBLG solution during crystallization. Figure B was obtained after Sénarmont's compensation of Figure A. The fibrillar character of the superstructure was revealed by stretching. The main axis of the fibrous structure was positioned parallel to the analyser's azimuth (long arrow), showing the wave-like banded aspect of the birefringence image (A). The helical intertwisted arrangement of the cords is evident. Three cords merge in a helical fashion (white arrows) and thinner fibers depict an undulated appearance. The smaller, thinner, arrows at 45 degrees from the polarizer indicate the relative position of the compensated positive birefringence of molecules in alternate bands. All components at 90 degrees from the dark compensated bands are brightly birefringent. Comparison of Figures A and B allows the observation of various helicity degrees (Twisted Grain Boundary). Bar, $20 \mu \mathrm{m}$.

\section{Acknowledgments}

This investigation was supported by grants of Brazilian research agencies CNPq and FAPESP.

\section{References}

1. Akagi, K.; Piao, G.; Kaneko, S.; Sakamaki, K.; Shirakawa, H.; Kyotani, M. Science, v. 282, p. 1683$1686,1998$.
2. Athenstaedt, H. Ann. N.Y. Acad. Sci. v. 238, p. 7-25, 1974.

3. Cornelissen, J.J.L.M.; Fisher, M.; Sommerdick, N.A.J.M.; Nolte, R.J.M. Science, v. 280, p. 14271430, 1998

4. Gupta, V.K.; Skaife, J.J.; Dubrovsky, T.B.; Abbot, N.L. Science, v. 279, p. 2077-2080, 1998.

5. Haller, I. J. Am. Chem. Soc., v. 100, p. 8050-8055, 1978. 
6. Jenekhe, S.A.; Chen, X.L. Science, v. 279, p. 19031906, 1998.

7. Whitesell, J.K.; Chang, H.K. Science, v. 261, p. 73-76, 1993.

8. Drzaic, P.S. Liq. Cryst., v. 26, p. 623-627, 1999.

9. Heppke, G.; Moro, D., Science, v. 279, p. 1872-1873, 1998.

10. Baranauskas, V.; Vidal, B.C.; Parizotto, N.A. Appl. Biochem. Biotech., v. 69, p. 91-96, 1998.

11. Vidal, B.C.; Baranauskas, V.; Parizotto, N.A. $5^{\text {th }} I n$ ternational Congress on Cell Biology, Madrid, p. 64, July/1992.

12. Fukada, E., Ann. N.Y. Acad. Sci., v. 238, p. 7-24, 1974.

13. Jaworeck, T.; Neher, D.; Wegner, G.; Wiringa, R.H.; Schouten, A.J. Science, v. 279, p. 57-60, 1998.

14. Vidal, B.C. Cell. mol. Biol., v. 32, p. 109-112, 1986.

15. Vidal, B.C. C.R. Acad. Sci. Paris, Life sciences, v. 318, p. 173-178, 1995.

16. Vidal, B.C. C.R. Acad. Sci. Paris, Life sciences, v. 318, p. 831-836, 1995.
17. Hartshorne, N.H. The Microscopy of Liquid Crystals, Microscope Publications Ltd., London, 1974.

18. Robinson, C. Tetrahedron, v. 13, p. 219-234, 1961.

19. Livolant, F. J. Phys., v. 48, p. 1051-1066, 1987.

20. Vidal, B.C. Histochemie, v. 30, p. 102-107, 1972.

21.Goodby, J.W.; Waugh, M.A.; Stein, S.M.; Chin, E.; Pindak, R.; Patel, J.S. Nature, v. 337, p. 449-452, 19899

22. Isaert, N.; Navailles, L.; Barois, P.; Nguyen, H.T. J. Phys. II France, v. 4, p. 1501-1518, 1994.

23. Li, M-N.; Nguyen, H.T.; Sigaud, G. Liq. Cryst., v. 20, p. 361-365, 1996.

24.Cassim, J.Y.; Taylor, E.W. Biophys. J., v. 5, p. $532-$ $552,1965$.

25.Cassim, J.Y.; Tobias, P.S.; Taylor, E.W. Biochim. Biophys. Acta, v. 168, p. 463-471, 1968.

26. DeVoe, H. J. Chem. Phys., v. 41, p. 393-400, 1964.

27. DeVoe, H. J. Chem. Phys. v. 43, p. 3199-3208, 1965.

28. Radcliffe, M.; Brostrom, M.L.; Epstein, K.A.; Rappaport, A.G.; Thomas, B.N.; Shao, R.; Clark, N.A. Liq. Cryst., v. 26, p. 789-794, 1999.

29. Kiss, G.; Porter, R.S. Mol. Cryst. Liq. Cryst., v. 60, p. 267-280, 1980.

FAPESP helped in meeting the publication costs of this article 